Journal of Case Reports 2018;8(2):91-94

\title{
Foreign Body Ingestion in a Patient with Psychiatric Illness
}

\author{
Tulit Chhabra, Satish Dalal, Mahavir Singh, Chisel Bhatia, Jagat Pal Beniwal \\ Department of General Surgery, Pt. B. D. Sharma Postgraduate Institute of Medical Sciences (P.G.I.M.S.), Rohtak-124001, Haryana, \\ India.
}

\section{Corresponding Author:}

Dr. Chisel Bhatia

Email: chiselbhatia@gmail.com

This is an Open Access article distributed under the terms of the Creative Commons Attribution License (creativecommons.org) licenses/by/3.0).

\begin{abstract}
Background: There are many challenges in treating patients who engage in deliberate foreign-body ingestion as a method of self-harm. Whereas other forms of mutilation may be dangerous to patients and distressing to their treaters, foreign-body ingestion carries a sense of insidiousness in the lack of outward evidence that harm has been done and in the latent risk of further injury even after a diagnosis has been made. Case Report: Here we report a case of foreign body ingestion in a patient with psychiatric illness and discuss what is known about deliberate foreign-body ingestion and some of the clinical problems they pose, with a focus on medical literature regarding the diagnosis and treatment of such cases. Conclusion: Timely counseling and psychiatric support is essential for foreign body ingestions.
\end{abstract}

Keywords: Endoscopy, Counseling, Foreign Bodies, Risk, Self-Injurious Behavior.

\section{Introduction}

Foreign body ingestion is most common among children aged 6 months to 6 years, but frequently seen among adults too. Ingestion of true foreign bodies occurs more frequently in children which includes coins, marbles, button tops but batteries, safety pins [1].

Besides esophageal food bolus ingestion, true foreign body ingestion in adults is more common in patients with psychiatric disorders, alcohol intoxication and prisoners seeking secondary gain and elderly population [2]. Most ingested foreign bodies (80\%-90\%) pass spontaneously. However, approximately 10\%-20\% cases of foreign body ingestion require endoscopic removal, while less than $1 \%$ will need surgery for foreign body extraction or to treat complications [3]. Inspite of the mostly benign natural course, ingestion of foreign bodies is associated with increased morbidity. Especially, care of patients who engage in repeated acts of deliberate selfharm can prove costly, both economically and in terms of the morale of their care providers. This case report aims to provide comprehensive review of diagnostic and therapeutic treatment options for swallowed foreign bodies in adults.

\section{Case Report}

A 43-year old, male patient suffering from major depression was brought to emergency department by his wife. Patient had ingested sharp end of knife after separating it from handle with the intention of committing suicide, a night before. At the time of admission, patient was asymptomatic and his general condition was good, arterial blood pressure (BP) was measured as 120/80 $\mathrm{mmHg}$ and pulse was $78 / \mathrm{min}$. There was no history of respiratory discomfort, epigastric pain, nausea and vomiting. On physical examination, both hemi-thorax were moving equally, no tachypnea, no pathological sounds were audible and the examination of cardiovascular system was normal. Abdominal examination revealed all quadrants moving equally with respiration and on palpation no tenderness was present in any quadrant. Laboratory parameters 
were as follows: CRP: normal, hemoglobin: 9.7

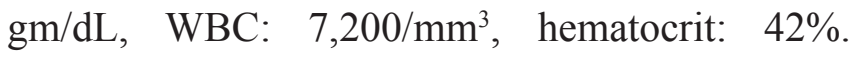
Plain abdominal X-ray antero-posterior view [Fig.1] showed a foreign body in the stomach at the level of pylorus measuring about $5 \times 2 \mathrm{~cm}$ with sharp edges. No sub-diaphragmatic free air was present. Upper gastrointestinal (GI) endoscopy had failed to endoscopically extirpate the foreign body. After endoscopic failure, the patient was hospitalized and kept under observation. During hospitalization, patient complained of nausea and pain in epigastric area. Since the knife was present at same position even after two days, as seen in repeat X-ray abdomen, surgical intervention was planned. Laprotomy (gastrotomy) was performed and foreign body was retrieved. Patient was discharged after good recovery with normal vital signs and physical examination findings on $3^{\text {rd }}$ post-operative day. Before discharge his psychiatric evaluation was carried out and the required antipsychotic treatment was started by the psychiatrist.

\section{Discussion}

Older children and non-impaired adults may identify the ingestion and localize discomfort whereas, mentally impaired adults and those with psychiatric illness, a medical evaluation may be difficult and may thus present with choking, refusal to eat, vomiting, drooling, wheezing, blood-stained saliva or respiratory distress. Impaction, perforation, or obstruction often occurs at GI angulations or narrowing. Hence, patients with previous GI tract surgery or congenital gut malformations are at increased risk. This means that the type of diagnostic evaluation and the extent and urgency of a possible intervention are decided on the basis of the information gained about the ingested foreign body, subjective complaints, and the clinical findings [4].

A large number of foreign bodies have been described in literature depending upon their size, surface, consistency etc. and also on the basis of whether they are radio-opaque or not on radiograph

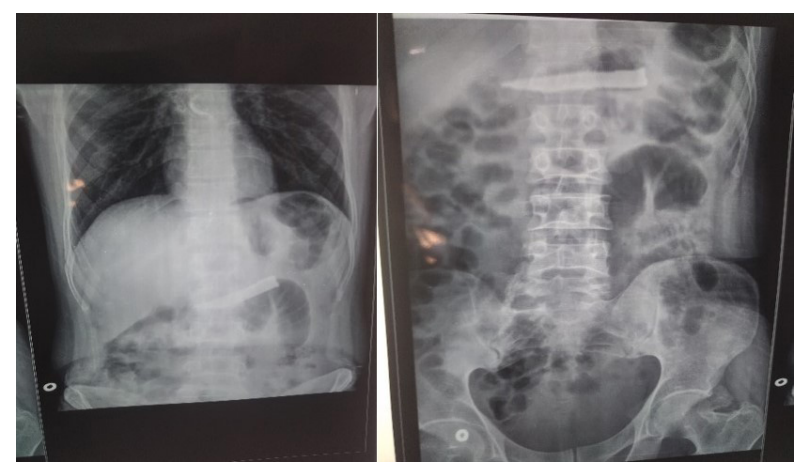

Fig.1: Plain abdominal AP radiograph shows foreign body in the stomach at the level of pylorus measuring about $5 \times 2 \mathrm{~cm}$ with sharp edges.

[5]. When a history of foreign body ingestion is elicited, a radiographic evaluation of the neck, chest and abdomen is recommended to assess the presence, location, size, configuration, and number of ingested objects. To minimize exposure to radiation, plain radiography is recommended as the initial screening method, but false-negative rates are as high as $47 \%$. Therefore, bi-planar radiography is recommended if the object is not detected on plain radiographs. Most true foreign bodies can be identified radiographically, however in case of nonradiodense and some radiodense foreign bodies, native $\mathrm{x}$-ray examination may not be sufficient to exclude ingestion of a foreign body [6]. Ngan et al . showed a sensitivity of only $32 \%$ and a specificity of $91 \%$ for ingested fish bones in native x-ray films of 354 patients [7]. Small foreign bodies, may be concealed by fluids and soft tissue masses. Such foreign bodies can be excellently identified by using computed tomography (CT) scanning, with a sensitivity of $100 \%$ and a specificity of $91 \%$. Thus, CT has an important role in the diagnostic evaluation of ingested foreign bodies. It not only provides better anatomic information, but can also detect other complications such as abscess formation, mediastinitis, or aortic/tracheal fistulas [8]. In the setting of radiological diagnostic evaluation, use of a barium swallow is not recommended because of the risk of aspiration and because coating of the foreign body and esophageal mucosa with contrast 
interferes with endoscopic visualization [5]. The natural course after ingestion of a foreign body is asymptomatic in $80 \%$ of cases where the foreign body passes uneventfully. Endoscopic intervention is indicated in about $20 \%$ of cases and surgery is required in less than $1 \%$ of cases [9].

Conservative outpatient management by means of clinical observation is appropriate for asymptomatic patients with blunt objects in the stomach that are smaller than $2-2.5 \mathrm{~cm}$ in diameter and $5-6 \mathrm{~cm}$ in length. Patients should be instructed to be aware of signs of perforation or small-bowel obstruction and to observe their stools continuously. In the absence of symptoms, weekly radiographs are sufficient to document the progression of the foreign body. If the foreign body fails to pass beyond the stomach within 3-4 weeks, it should be extracted endoscopically. The failure rate of the conservative approach is $2 \%-5 \%$ and it is always advisable in asymptomatic individuals [5].

Esophageal foreign objects and food bolus impacted in the esophagus should be removed within 24 hours because delay decreases the likelihood of successful removal and increases the risk of complications [5]. Though endoscopy is the preferred method for handling foreign body ingestion in the GI and respiratory tracts, but when a patient presents with secondary complications to asymptomatic foreign body ingestion, young children and patients with psychiatric illness it is hard to establish a clear diagnosis, a surgical exploration will determine diagnosis and treatment both. Surgery is the best therapeutic procedure for failed endoscopic procedure, multiple foreign bodies, and complications (perforation, abscess etc). Cervicotomy, thoracotomy and laparotomy are the options when there is a visceral rupture, infection or hemodynamic compromise. In recent years, laparoscopy has emerged as an accurate procedure for visual evaluation of the intraabdominal cavity [10].

Removal of foreign body from stomach is controversial. Management of ingested foreign body varies according to type, size and location. In our patient, surgical intervention was required as endoscopic removal was unsuccessful and ingested object was sharp which increases risk of perforation. Delayed presentation and silent perforations of stomach caused by foreign bodies has also been reported. Therefore, removal of long sharp objects longer than $3 \mathrm{~cm}$ is recommended even in asymptomatic patients [11].

The literature on personality disorders covers a dazzling array of self-injurious behaviors, objects not just swallowed but inserted into the abdomen, heart, airway, bladder, breast, ear, legs, and arms, even into the cranium [12]. Our case report reflects the characteristics of such patients as self-injurious. Internal feelings of emptiness and unbearable tension often precede to such behavior. Our report emphasizes the importance of psychiatric support that should be given to these patients as such behavior is repeated in large number of patients.

\section{Conclusion}

Foreign body ingestion with its insidious nature may have a complicated course, which may cause serious complications when not correctly managed. Though majority of them can be managed conservatively, a close monitoring is required by a surgeon and an endoscopist to avoid complications associated with foreign body ingestion. Timely counseling and psychiatric support is essential for proper management of such cases.

Contributors: TC, CB: manuscript writing, patient management; SD, MS: manuscript editing, patient management; JPB: critical inputs into the manuscript, patient management. CB will act as guarantor. All authors approved the final version of this manuscript.

Funding: None; Competing interests: None stated.

\section{References}

1. Shivkumar AM, Naik AS, Prashanth KB, Hongal GF, Chaturvedy G. Foreign bodies in upper digestive tract. Indian J Otolaryngol Head Neck Surg. 2006;58:63-68. 
2. Ambe P, Weber SA, Schauer M, Knoefel WT. Swallowed foreign bodies in adults. Dtsch Arztebl Int. 2012;109:869-875.

3. Dray X, Cattan P. Foreign bodies and caustic lesions. Best Pract Res Clin Gastroenterol. 2013;27:679-689.

4. Ikenberry SO, Jue TL, Andersen MA, Appalaneni V, Banerjee S, Menachem TB, et al. ASGE standards of practice committee. Management of ingested foreign bodies and food impactions. Gastrointest Endosc. 2011;73:1085-1091.

5. Birk M, Bauerfield P, Deprez PH, Hafner M, Hartmann $\mathrm{D}$, Hassan $\mathrm{C}$, et al. Removal of foreign bodies in the upper gastrointestinal tract in adults; European Society of Gastrointestinal Endoscopy (ESGE) Clinical guidelines. Endoscopy. 2016;48:489-496.

6. Pfau PR. Removal and management of esophageal foreign bodies. Tech Gastrointest Endosc. 2014;16:3239.

7. Ngan JH, Fok PJ, Lai EC, Branicki FJ, Wong J. A prospective study on fish bone ingestion. Experience of 358 patients. Ann Surg. 1990;211:459-462.

8. Marco De Lucas E, Sadaba P, Lastra Garcia-Baron P, Ruiz Delgado ML, Gonzalez SL, Ortiz A. Value of helical computed tomography in the management of upper esophageal foreign bodies. Acta Radiol. 2004;45:369374.

9. Zhang S, Cui Y, Gong X R, Gu F, Zhong B. Endoscopic management of foreign bodies in the upper gastrointestinal tract in South China: a retrospective study of 561 cases. Dig Dis Sci. 2010;55:1305-1312.

10. Arango LAA, Sierra LPL, Gutieerez DCM, Grisales MJ. Incidental foreign body in the gastrointestinal tract. Report of three cases and literature review. Rev Col Gastroenterol. 2011;26:308-318.

11. Wishner JD, Rogers AM. Laparoscopic removal of a swallowed toothbrush. Surg Endosc. 1997;11:472-473.

12. Green AH. Self-destructive behavior in battered children. Am J Psychiatry. 1978;135:579-582. 